
\title{
R Research Soure \\ Dependence of Capacitive Properties of an EDLC on Exfoliation Time of Graphite Electrodes
}

\section{Dinithi S. K. Rajaguru}

Wayamba University of Sri Lanka

Kamal Vidanapathirana ( $\nabla$ kamalpv41965@gmail.com )

Wayamba University of Sri Lanka https://orcid.org/0000-0003-0676-7627

Kumudu S. Perera

Wayamba University of Sri Lanka

\section{Research Article}

Keywords: Liquid phase exfoliation, exfoliated graphite, electrochemical impedance spectroscopy, cyclic voltammetry, galvanostatic charge discharge

Posted Date: March 19th, 2021

DOI: https://doi.org/10.21203/rs.3.rs-298632/v1

License: @ (i) This work is licensed under a Creative Commons Attribution 4.0 International License. Read Full License 


\title{
Dependence of capacitive properties of an EDLC on exfoliation time of graphite electrodes
}

\author{
Dinithi S. K. Rajaguru, Kamal P. Vidanapathirana*, Kumudu S. Perera
}

Polymer Electronics Research Group, Department of Electronics, Wayamba University of Sri Lanka, Kuliyapitiya, Sri Lanka

$\begin{array}{lll}* \text { * Corresponding author: } & \text { Email } & -\underline{\text { kamalpv@wyb.ac.lk }} \\ & \text { ORCID ID } & - \text { https://orcid.org/0000-0003-0676-7627 }\end{array}$




\begin{abstract}
The scientific focus has been directed through the production and application of 'wonder material- graphene' after its discovery in 2004. But the mass production cost has become a huge disadvantage towards commercializing graphene based manufactures. As alternative low cost material, exfoliated graphite (EG) has emerged to be a novel nanostructured carbon material with a potential for electrochemical energy storage device applications owed to its unique characteristics similar to graphene. In this study a series of EG samples were prepared by a surfactant mediated liquid phase exfoliation method by changing the exfoliation time. Electrochemical double layer capacitors (EDLCs) were fabricated using different EG samples as an electrode material and a gel polymer electrolyte (GPE). They were characterized by electrochemical impedance spectroscopy (EIS), cyclic voltammetry (CV) and galvanostatic charge discharge (GCD) techniques. EDLC having EG electrodes of $10 \mathrm{~h}$ exfoliation time showed the highest results with single electrode specific capacitance $\left(\mathrm{C}_{s c}\right)$ of $4.12 \mathrm{~F} \mathrm{~g}^{-1}$, single electrode specific discharge capacitance $\left(\mathrm{C}_{s d}\right)$ of $1.10 \mathrm{~F} \mathrm{~g}^{-1}$ and relaxation time of $0.22 \mathrm{~s}$ from $\mathrm{CV}$, GCD and EIS respectively.
\end{abstract}

Keywords: Liquid phase exfoliation, exfoliated graphite, electrochemical impedance spectroscopy, cyclic voltammetry, galvanostatic charge discharge

\title{
Declarations
}

Funding - This study was funded by the National Research Council of Sri Lanka under the grant number NRC-18-007

Conflicts of interest/Competing interests (include appropriate disclosures)

Availability of data and material (data transparency)

Code availability - Not applicable

Authors' contributions (optional: please review the submission guidelines from the journal whether statements are mandatory)

\section{Relevance Summary}

* Exfoliated graphite has been identified as a more suitable electrode material for EDLCs.

* In this study, exfoliated graphite electrodes were prepared using a low cost exfoliation time.

* Variation of capacitive behavior was investigated by varying the exfoliation time.

* It was possible to observe that exfoliation time, $\mathrm{t}$ is a determinant factor of the specific capacitance.

* However, it has a limitation over controlling the capacitance. ie. only at a particular value of $t$, specific capacitance reaches the maximum state.

\section{Introduction}

Exfoliated graphite (EG) denotes to graphite which has a degree of separation of carbon layers in the graphite. 'Exfoliation' is the process by which the separation occurs [1]. Several methods have been deployed to achieve graphite exfoliation, each with benefits and drawbacks [2]. Most commonly used methods include, mechanical exfoliation, chemical conversion, thermal exfoliation, electrochemical exfoliation and liquid phase exfoliation. Liquid phase exfoliation is among the most inexpensive and eco-friendly processes for EG production. Graphite can 
be successfully exfoliated in liquid mediums by exploiting ultrasound to extract layers. The process comprises three main steps: dispersion of graphite in a solvent, exfoliation and purification [3].

Liquid phase exfoliation can be performed in various mediums such as organic solvents, aqueous surfactant solutions and ionic liquids [4]. Organic solvents like 1-methyl-2-pyrrolidinone (NMP), g-butyrolactone (GBL), N, N-dimethylacetamide (DMA), N-vinyl-2-pyrrolidone (NVP) and N, N-dimethylformamide (DMF) have surface energies similar to graphene. But the toxicity and high cost limited their use in mass production. Exfoliation of graphite in water with the aid of surfactants can avoid such complications. Surfactants support EG to remain suspended in aqueous mediums while providing a suitable solution for hydrophobic nature of EG sheets. Lotya $e t$ al. reported a surfactant mediated liquid phase exfoliation using sodium dodecylbenzenesulfonate (SDBS) as surfactant [5]. Since then, a variety of cationic, anionic and non-ionic surfactants have been used. Nawaz et al. evaluated the effect of concentration of sodium cholate (SC) on the exfoliation of graphite in aqueous media [6]. These surfactants are not only used as intercalating agents but also as stabilizers to prevent EG from restacking in the solution.

EG has a higher volume than graphite due to porosity which occurs during local separation. And also, it exhibits ultra-light weight and high selection sorption capacity [7]. There are numerous research works demonstrating EG structure to be different, like a fluffy cellular structure or a worm-like structure, depending on its exfoliation degree [8]. Because of its uncommon structure, EG exhibits high specific surface area, improved porous structure, low density, high concentration of edges and surface defects and high thermal and chemical stability. These properties of EG that differ from conventional graphite have led to several applications in batteries and supercapacitors. EG and EG-based materials have been explored for use as electrode materials in practical applications, owing to their pristine and highly conducting nature [4].

Properties of EG will vary upon its synthesis method. Variables such as, exfoliation time, surfactant type, surfactant concentration and solvent will affect on the physical and chemical characteristics of EG. Considerable deviation of these factors can have a huge impact on the characteristic features of the devices that are fabricated with EG.

Most of the research works are based on commercially available graphite and/or graphene based supercapacitors while few studies are based on EG based supercapacitors. Out of previous studies, many are reported with liquid electrolytes [9-11]. This study mainly focuses on the surfactant mediated liquid phase exfoliation of Sri Lankan natural graphite and to use the resulting EG as an electrode material in an EDLC with a GPE while giving an estimation on the capacitance of the EG based EDLCs through a viewpoint of exfoliation time.

\section{Experimental}

\subsection{Materials}

Sri Lankan natural graphite was obtained from Bogala Graphite Lanka Ltd, Bogala, Sri Lanka. Sodium dodecylbenzenesulfonate (SDBS), polyvynilidene fluoride (PVDF), zinc trifluoromethanesulfonate $\left(\mathrm{Zn}\left(\mathrm{CF}_{3} \mathrm{SO}_{3}\right)_{2}\right.$, $\mathrm{ZnTF}$ ), ethylene carbonate (EC), propylene carbonate (PC) and 1-methyl-2-pyrrolidinone were purchased from Sigma Aldrich and used without further purification. 


\subsection{Preparation of Exfoliated graphite (EG) electrodes}

Graphite was exfoliated in aqueous SDBS solution $\left(5 \mathrm{mg} \mathrm{ml}^{-1}\right)$ using ultrasonic homogenizer (Athena ATP 150) for different time periods (45 min, 2, 5, 10, $15 \mathrm{hrs)}$ ) and filtered separately. The filtered EG samples were then dried and mixed with 1-methyl-2-pyrrolidinone before deposited onto fluorine-doped tin oxide (FTO) glasses using the doctor blade method as the electrodes. After coating, electrodes were dried to evaporate the solvent and also to improve the contacts. Area of the electrode was $1 \mathrm{~cm}^{2}$.

\subsection{Preparation of gel polymer electrolyte (GPE)}

Required amounts of PVDF, EC, PC and ZnTF were used to prepare a GPE as reported in our previous study [12]. The mixture was stirred well and heated at $130{ }^{\circ} \mathrm{C}$. The resultant hot mixture was pressed in between two wellcleaned glass plates. Upon cooling, it was possible to obtain a bubble-free thin film.

\subsection{Fabrication of EDLCs}

The EDLC was fabricated with a pair of EG electrodes and with the GPE of the configuration, EG/GPE/EG. Several EDLCs were fabricated using electrodes prepared with different exfoliation time.

\subsection{Characterization}

\subsubsection{Electrochemical Impedance Spectroscopy (EIS)}

EDLCs fabricated with different electrodes were initially tested with Electrochemical Impedance Spectroscopy (EIS) technique using an impedance analyzer (Metrohm Autolab M101) at room temperature. Impedance data were gathered in the frequency range $400 \mathrm{kHz}-0.01 \mathrm{~Hz}$. Nyquist plots were drawn from the impedance data. The real part of the complex capacitance $\left(\mathrm{C}^{\prime}{ }_{(\omega)}\right)$ and the imaginary part of the complex capacitance $\left(\mathrm{C}^{\prime \prime}{ }_{(\omega)}\right)$ were calculated based on the real $\left(\mathrm{Z}_{(\omega)}^{\prime}\right)$ and imaginary $\left(\mathrm{Z}_{(\omega)}^{\prime \prime}\right)$ parts of the impedance and Bode plots were drawn [13].

\subsubsection{Cyclic Voltammetry (CV)}

For the cyclic voltammetry (CV), a three electrode electrochemical cell was used where one electrode of the EDLC was used as the working electrode and the other one as the reference and counter electrodes. Cycling was done using a computer controlled potentiostat (Metrohm Autolab M101). In one of our previous studies, we have found that the potential window within which the EDLC could operate without any distortion was as -1.0 to $1.0 \mathrm{~V}$ [14]. Same potential window was selected for the present study. The scan rate selected was $10 \mathrm{mVs}^{-1}$. The effect of exfoliation time on single electrode specific capacitance $\left(\mathrm{C}_{s c}\right)$ was determined by cycling EDLCs having EG electrodes with different exfoliation times. $\mathrm{C}_{s c}$ was calculated using the equation 1.

$$
C_{s c}=\frac{2 \int I d V}{m S \Delta V}
$$

In the equation $1, \int I d V$ is the integrated area of the cyclic voltammogram, $m$ is the mass of a single electrode, $\Delta V$ is width of the potential window and $S$ is the scan rate [15]. The optimum exfoliation time was determined considering 
the $\mathrm{C}_{s c}$ values. To observe the durability of the device, continuous cycling was then carried out for all EDLCs for 500 cycles.

\subsubsection{Galvanostatic charge discharge (GCD) test}

Galvanostatic charge discharge (GCD) test was carried out within the potential window, 0.1 to $1.0 \mathrm{~V}$ at a constant current, $I$ of $0.05 \mathrm{~mA}$ using a Metrohm M101 potentiostat. Single electrode specific discharge capacitance, $C_{s d}$ was calculated using the equation 2 .

$$
C_{s d}=\frac{I}{m \frac{d V}{d t}}
$$

where, $m$ is the mass of an electrode, $d V / d t$ is the rate of potential drop excluding internal resistance [16].

\section{Results and discussion}

\subsection{Preparation of exfoliated graphite (EG) electrodes}

In the exfoliation process, when graphite powder is mixed in aqueous SDBS solution, a graphitic collidal suspension is formed. An uniform suspension is obtained mainly due to surfactant nature of SDBS. Under the ultra-sonication, the solvated ions $\left(\mathrm{Na}^{+}\right.$and $\left.\mathrm{DBS}^{-}\right)$will be co-intercalated into graphite layers. This intercalation permits to induce expansion of the graphite interlayers while weakening the van der waals forces between graphitic layers. When there are sufficient amount of intercalated ions, the inflated graphite will generate ultimate expansion enabling separation of the graphitic layers (fig.1) [17]. Ultimately, it is possible to obtain an EG suspension consists of single (graphene) and multilayer sheets [18]. When the exfoliation time increases, the number of collisions between graphitic particles increases while giving more time for layer seperation. Once the exfoliation time rises, the exfoliated suspension may contain graphene and few layer EG.

Fig. 1: Schematic illustration for mechanism of surfactant mediated liquid phase exfoliation of graphite

\subsection{Electrochemical Impedance Spectroscopy (EIS)}

From EIS tests, properties of the electrolyte and the electrodes of an EDLC can be identified through Nyquist plots obtained at different frequencies. Mainly, three regions are defined in the Nyquist plots. High, mid and low frequency regions are related with the properties of bulk electrolyte, electrode-electrolyte interface and capacitive behavior of an EDLC respectively.

Nyquist plots of all EDLCs mainly consist of a semi-circle at mid frequency area followed by a tilted vertical line in the low-frequency region as depicted in fig. 2. Insert of fig. 2 illustrates the high frequency region. Ideal capacitance is represented by a vertical line parallel to imaginary axis $\left(-Z^{\prime \prime}\right)$. However, the tilted line suggests weaker capacitive features of the EDLC. This might arise due to problems of electrode surfaces as well as non uniform nature of electrodes. Semicircle in the mid frequency range occurs due to the charge transfer resistance at the electrode- 
electrolyte interface. Smaller semi-circle radius/diameter implies low charge transfer resistance [19]. Absence of a semi-circle that represent bulk electrolyte resistance at high frequency region might be due to the unavailability of required frequency values. When the exfoliation time increases the number of EG layers decreases creating more voids thus increasing the surface area. But at the same time, rather than separating into fewer layers, breaking into smaller pieces without decreasing the number of layers could happen. This decreases the accessibility of ions on the EG electrode surface. Therefore two main outcomes can be highlighted as the effect of varying exfoliation time: (i) separation of graphitic layers into fewer EG layers creating more spaces (pores) (ii) breaking of stacked graphitic sheets into small pieces (weaken the approachability for ions to inner layers) [20]. Combination of these two aspects influences the capacitive features of the EDLCs.

The Nyquist plots obtained for EDLCs having EG electrodes with different exfoliation time is shown in figure 2. EDLC with the EG electrodes exfoliated for $10 \mathrm{~h}$ gives the smallest semi-circle radius. Smallest value implies lower charge transfer resistance at the electrode-electrolyte interface. Hence, the storage of charges should be higher in that EDLC. At low frequency region, tilted lines are present in the EDLCs with EG electrodes having exfoliated time higher than $2 \mathrm{~h}$. In addition, they are almost parallel to each other. Absence of vertical lines may be due to problems of the electrodes. But, they all have capacitive features to some extent. This highlights the fact that with higher exfoliation time periods, EDLC tend to show capacitive features. Under lower exfoliation times, formation of EG may not take place resulting no noticeable capacitance.

Fig. 2: Nyquist plots obtained for EDLCs having EG electrodes with different exfoliation time

Figs. $3 \mathrm{a}$ and $3 \mathrm{~b}$ show the resulting Bode plots.

Fig. 3: Bode plots obtained for different EG samples (a) $\mathrm{C}^{\prime}$ versus frequency (in logarithmic scale) (b) $\mathrm{C}^{\prime \prime}$ versus frequency (in logarithmic scale).

The highest $\mathrm{C}_{s c}$ can be found using maximum $\mathrm{C}^{\prime}$ in fig. 3a. Values are given in table 1.

Table 1: Single electrode specific capacitance $\left(\mathrm{C}_{s c}\right)$, frequency where the maximum $\mathrm{C}^{\prime \prime}$ occur $\left(f_{0}\right)$ and relaxation time $\left(\tau_{0}\right)$ for the different EG electrodes.

\begin{tabular}{|c|c|c|c|}
\hline Exfoliation time & $\mathrm{C}_{s c}\left(\mathrm{~F} \mathrm{~g}^{-1}\right)$ & $f_{0}(\mathrm{~Hz})$ & $\tau_{0}(\mathrm{~s})$ \\
\hline 0 & 0.30 & 0.02 & 7.79 \\
\hline $45 \mathrm{~min}$ & 0.41 & 0.04 & 3.81 \\
\hline $2 \mathrm{~h}$ & 0.55 & 0.17 & 0.91 \\
\hline $5 \mathrm{~h}$ & 0.54 & 0.36 & 0.45 \\
\hline $10 \mathrm{~h}$ & 0.82 & 0.73 & 0.22 \\
\hline $15 \mathrm{~h}$ & 0.73 & 0.37 & 0.43 \\
\hline
\end{tabular}


According to the values, it is seen that the EDLC having the EG electrodes with an exfoliation time of $10 \mathrm{~h}$ has the optimum $\mathrm{C}_{s c}$. This well confirms that there is an effect from the exfoliation time on $\mathrm{C}_{s c}$. Upon increasing exfoliation time, more and more single EG layers may be formed favoring capacitive features. After a certain exfoliation time ( $10 \mathrm{~h}$ in the present study) exceeds, stacked graphitic layers may break into small pieces hindering the entry of ions into inner layers resulting a decrease in the capacity [21].

Another important parameter is the relaxation time $\left(\tau_{0}\right)$ which is the quantitative measurement of the response time of the EDLC. $\tau_{0}$ can be calculated by analyzing the Bode plots (fig. $3 \mathrm{~b}$ ) using the equation 3 [22].

$$
\tau_{0}=\frac{1}{2 \pi f_{0}}
$$

They are shown in Table 1 with the corresponding frequency values. Accordingly, EDLCs with the EG electrodes having an exfoliated time of $10 \mathrm{~h}$ shows the minimum $\tau_{0}$ further validating its higher capacitive properties.

\subsection{Cyclic Voltammetry (CV)}

The cyclic voltammograms obtained are shown in the fig. 4 . They all exhibited a parallelogram shape without any redox peaks implying their good capacitive behavior. This also suggests that there were no redox reactions and the charge storage mechanism was mostly based on the electrostatic mechanism. Thus, capacitive properties are solely due to ion accumulation instead of ion intercalation process. The highly reversible charge discharge process is confirmed by the almost mirror image symmetry around zero current line [23]. All the CV curves show a rapid current response on voltage reversal at each end potential. The single electrode specific capacitance $\left(\mathrm{C}_{s c}\right)$ was determined using the equation 1 . As per fig. 4 , area of the cyclic voltammograms with the exfoliation time till $10 \mathrm{~h}$ increases and decreases thereafter. This well agrees with the EIS results explained before.

Fig. 4: Cyclic voltammograms of EDLCs having EG electrodes with different exfoliation time. Potential window of $-1.0 \mathrm{~V}$ to $+1.0 \mathrm{~V}$ and scan rate $10 \mathrm{mV} \mathrm{s}^{-1}$. [(1) Graphite, (2) $45 \mathrm{~min} \mathrm{EG}$, (3) $2 \mathrm{~h} \mathrm{EG,} \mathrm{(4)} 5 \mathrm{~h} \mathrm{EG,} \mathrm{(5)} 10 \mathrm{~h} \mathrm{EG,} \mathrm{(6)} 15 \mathrm{~h}$ EG]

The stability and reversibility of any electrochemical device is of utmost importance from the practical point of view.

Fig. 5 demonstrates $\mathrm{C}_{s c}$ values of EDLCs for 500 cycles having EG electrodes with different exfoliation time.

Fig. 5: Single electrode specific capacitance $\left(C_{s c}\right)$ values obtained from continuous cycling over 500 cycles for EDLC with different EG electrodes. [(1) Graphite, (2) 45 min EG, (3) 2 h EG, (4) 5 h EG, (5) 10 h EG, (6) 15 h EG]

\subsection{Galvanostatic charge discharge (GCD) test}

Fig. 6 illustrates galvanostatic charge-discharge curves of EDLCs with different EG sample for first charge and discharge cycles. A rapid voltage drop was noticeable at the beginning of the discharging process for each electrode. This ohmic loss arises due to internal resistance of the device. 
Fig. 6: Initial charging and discharging cycles of different EG samples obtained in the $+0.1 \mathrm{~V}$ to $+1.0 \mathrm{~V}$ potential range. [(1) Graphite, (2) 45 min EG, (3) 2 h EG, (4) 5 h EG, (5) 10 h EG, (6) 15 h EG]

Fig. 7 displays the cycling stability of all EDLCs under constant current. EDLCs based on EG electrodes showed higher $\mathrm{C}_{s d}$ values than that of graphite. Those values increased upon increasing exfoliation time up to $10 \mathrm{~h}$ and then decreased with further increasing of exfoliation time. However, $\mathrm{C}_{s d}$ of all EDLCs displayed a slight up and down variation. Even under such variations, all EDLCs have been able to maintain a more or less constant $\mathrm{C}_{s d}$. This might be due to the property of the GPE which is called as self-healing property [24].

Fig. 7: Single electrode discharge capacitance $\left(\mathrm{C}_{s d}\right)$ values obtained from continuous charge-discharge cycling for 10000 cycles for different EG samples. [(1) Graphite, (2) 45 min EG, (3) 2 h EG, (4) 5 h EG, (5) 10 h EG, (6) 15 h EG]

\section{Conclusion}

EG samples with various exfoliation times were synthesized using a surfactant mediated liquid phase exfoliation. EDLCs were fabricated with the prepared EG samples. The electrochemical performances of these EDLCs were evaluated via EIS, CV and GCD techniques. EG sample with exfoliation time of 10 h showed maximum $\mathrm{C}_{s c}$ of 4.12 $\mathrm{F} \mathrm{g}^{-1}$ and maximum $\mathrm{C}_{s d}$ of $1.10 \mathrm{~F} \mathrm{~g}^{-1}$ by $\mathrm{CV}$ and $\mathrm{GCD}$ techniques respectively.

\section{Acknowledgements}

The authors gratefully acknowledge National Research Council (NRC grant 18-007) for the financial support and Bogala Graphite Lanka Ltd for proving samples.

\section{References}

[1] D. D. L. Chung, A review of exfoliated graphite. J. Mater. Sci. 51, pp. 554-568 (2015).

[2] M. Cai, D. Thorpe, D. H. Adamson, and H. C. Schniepp, Methods of graphite exfoliation. J. Mater. Chem. 22, pp. 24992-25002 (2012).

[3] A. Ciesielski and P. Samorì, Graphene via sonication assisted liquid-phase exfoliation. Chem. Soc. Rev. 43, pp. 381-398 (2014).

[4] W. Du, X. Jiang, and L. Zhu, From graphite to graphene: direct liquid-phase exfoliation of graphite to produce single- and few-layered pristine graphene. J. Mater. Chem. A 1, pp. 10592-10606 (2013).

[5] M. Lotya, Y. Hernandez, P. J. King, R. J. Smith, V. Nicolosi, L. S. Karlsson, F. M. Bighe, S. De, Z. Wang, I.T. MacGovern, G.S. Duesberg and J. N. Coleman, Liquid Phase Production of Graphene by Exfoliation of Graphite in Surfactant / Water Solutions. J. Am. Chem. Soc. 131, pp. 3611-3620 (2009).

[6] K. Nawaz, M. Ayub, M. B. Khan, A. Hussain, A. Q. Malik, M. B. K. Niazi, M. Hussain, A. U. Khan and N. Ul-Haq, Effect of concentration of surfactant on the exfoliation of graphite to graphene in aqueous media. Nanomater. Nanotechnol. 6, pp. 1-7 (2016). 
[7] J. S. Evans, T. Guo, Y. Sun, W. Liu, L. Peng, Z. Xu, C. Gao and S. He, Shape-controlled Tens-nanometersthick Graphite and Worm-like Graphite through Lithographic Exfoliation. Carbon. 135, pp. 248-252 (2018).

[8] X. Hong and D. D. L. Chung, Exfoliated graphite with relative dielectric constant reaching 360 , obtained by exfoliation of acid- intercalated graphite flakes without subsequent removal of the residual acidity. Carbon. 91, pp. 1-10 (2015).

[9] A. Ambrosi and M. Pumera, Electrochemically Exfoliated Graphene and Graphene Oxide for Energy Storage and Electrochemistry Applications. Chem. Eur. J. 22, pp. 153-159 (2016).

[10] Y. Song, T. Liu, G. Xu, D. Feng, B. Yao, T. Kuo, X. Liu and Y. Li, Tri-layered graphite foil for electrochemical capacitors. J. Mater. Chem. A 4, pp. 7683-7688 (2016).

[11] A. Raghunandanan, M. Yeddala, P. Padikassu, and R. Pitchai, Partially Exfoliated Graphite Paper as FreeStanding Electrode for Supercapacitors.ChemistrySelect. 3, pp. 5032-5039 (2018).

[12] W. A. S. S. Weerasinghe, K. P. Vidanapathirana, Y. M. C. D. Jayathilake, and K. S. Perera, Performance of an all polymer supercapacitor. Proceeding 8th Int. Res. Conf. KDU, pp. 49-52 (2015).

[13] C. Yang, C. Y. Vanessa Li, F. Li, and K.-Y. Chan, Complex Impedance with Transmission Line Model and Complex Capacitance Analysis of Ion Transport and Accumulation in Hierarchical Core-Shell Porous Carbons. J. Electrochem. Soc. 160, pp. H271-H278 (2013).

[14] D. S. K. Rajaguru, K. P. Vidanapathirana, and K. S. Perera, Exfoliated graphite as electrochemical double layer capacitor electrode: Electrochemical impedance study. Sri Lankan J. Phys. 22, pp. 20-28 (2021).

[15] C. M. Bandaranayake, Y. M. C. D. Yayathilake, K. S. Perera, K. P. Vidanapathirana, and L. R. A. K. Bandara, Investigation of a gel polymer electrolyte based on polyacrylonitrile and magnesium chloride for a redox capacitor. Ceylon J. Sci. 45, pp. 75-82 (2016).

[16] C. W. Liew and S. Ramesh, Comparing triflate and hexafluorophosphate anions of ionic liquids in polymer electrolytes for supercapacitor applications. Materials.7, pp. 4019-4033 (2014).

[17] Y. Zhang, Y. Xu, J. Zhu, L. Li, X. Du, and X. Sun, Electrochemically exfoliated high-yield graphene in ambient temperature molten salts and its application for flexible solid-state supercapacitors. Carbon. 127, pp. 392-403 (2018).

[18] K. Zhou, Y. Shi, S. Jiang, L. Song, Y. Hu, and Z. Gui, A facile liquid phase exfoliation method to prepare graphene sheets with different sizes expandable graphite. Mater. Res. Bull. 48, pp. 2985-2992 (2013).

[19] I. Yang, S. G. Kim, S. H. Kwon, M. S. Kim, and J. C. Jung, Relationships between pore size and charge transfer resistance of carbon aerogels for organic electric double-layer capacitor electrodes. Electrochim. Acta. 223, pp. 21-30 (2017).

[20] K. R. Paton, E. Varrla, C. Backes, R. J. Smith, U. Khan, A. O'Neill, C. Boland, M. Lotya, O. M. Istrate, P. King, T. Higgins, S. Barwich, P. May, P. Puczkarski, I. Ahmed, M. Moebius, H. Pettersson, E. Long, J. Coelho, S. E. O'Brien, E. K. McGuire, B. M. Sanchez, G. S. Duesberg, N. McEvoy, T. J. Pennycook, C. Downing, A. Crossley, V. Nicolosi and J. N. Coleman, Scalable production of large quantities of defect-free few-layer graphene by shear exfoliation in liquids. Nat. Mater. 13, pp. 624-630 (2014).

[21] M. Laipan, L. Xiang, J. Yu, B. R. Martin, R. Zhu, J. Zhu, H. He, A. Clearfield and L. Sun, Layered 
intercalation compounds: Mechanisms, new methodologies, and advanced applications. Prog. Mater. Sci. 109, pp. 100631-100728 (2020).

[22] J. P. Tey, M. A. Careem, M. A. Yarmo, and A. K. Arof, Durian shell-based activated carbon electrode for EDLCs. Ionics. 22, pp. 1209-1216 (2016).

[23] Y. Gao, Q. Yue, and B. Gao, High surface area and oxygen-enriched activated carbon synthesized from animal cellulose and evaluated in electric double-layer capacitors. RSC Adv. 5, pp. 31375-31383 (2015).

[24] X. Cheng, J. Pan, Y. Zhao, M. Liao, and H. Peng, Gel Polymer Electrolytes for Electrochemical Energy Storage. Adv. Energy Mater. 8, pp. 1-16 (2018). 
Figures

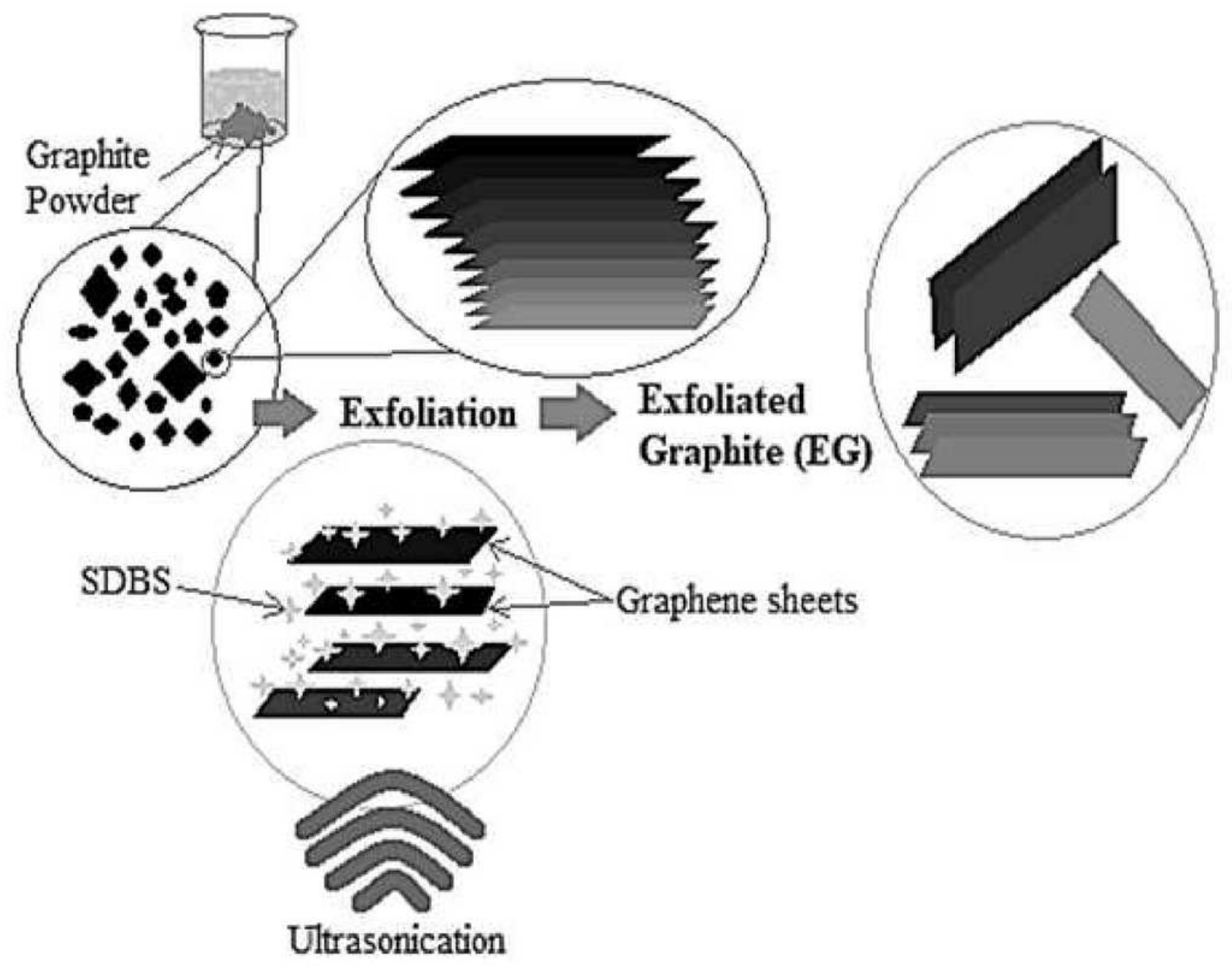

Figure 1

Schematic illustration for mechanism of surfactant mediated liquid phase exfoliation of graphite 


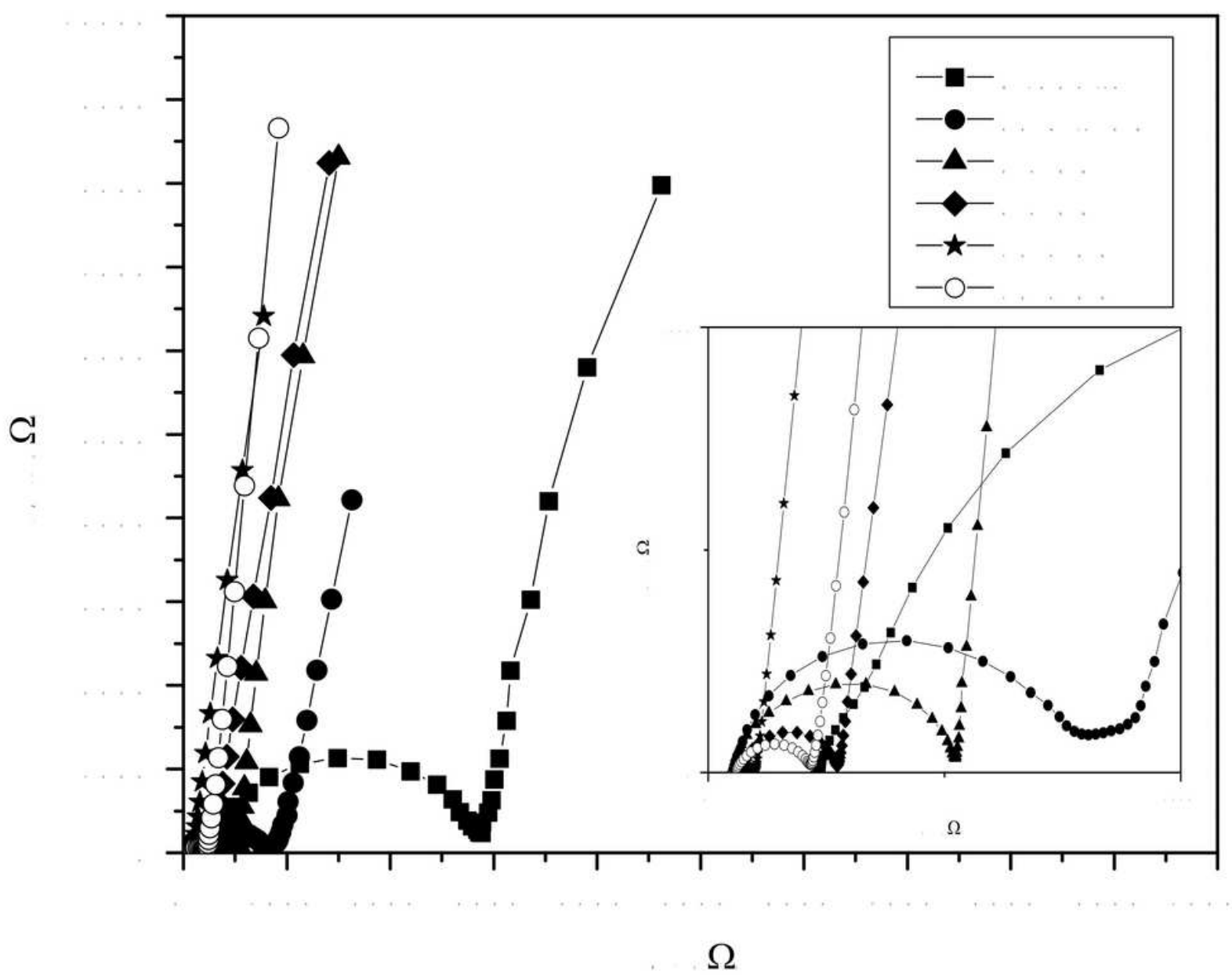

Figure 2

Nyquist plots obtained for EDLCs having EG electrodes with different exfoliation time 

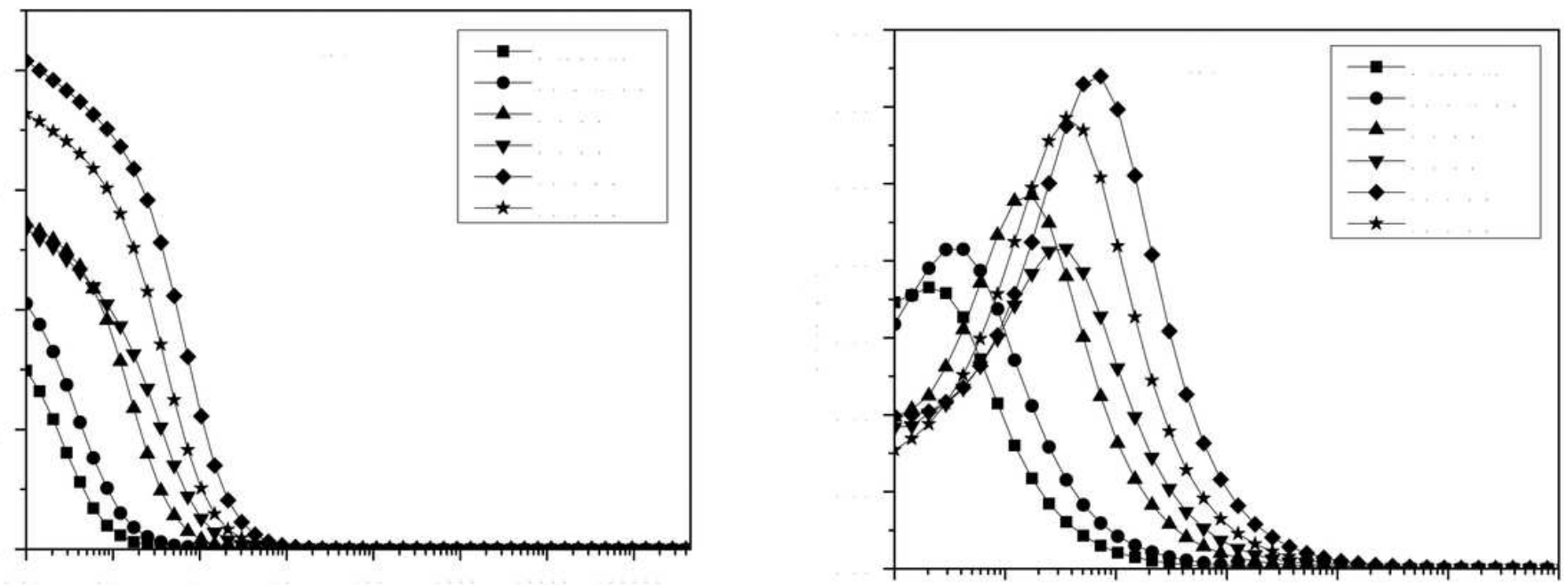

Figure 3

Bode plots obtained for different EG samples (a) C/ versus frequency (in logarithmic scale) (b) C// versus frequency (in logarithmic scale). 


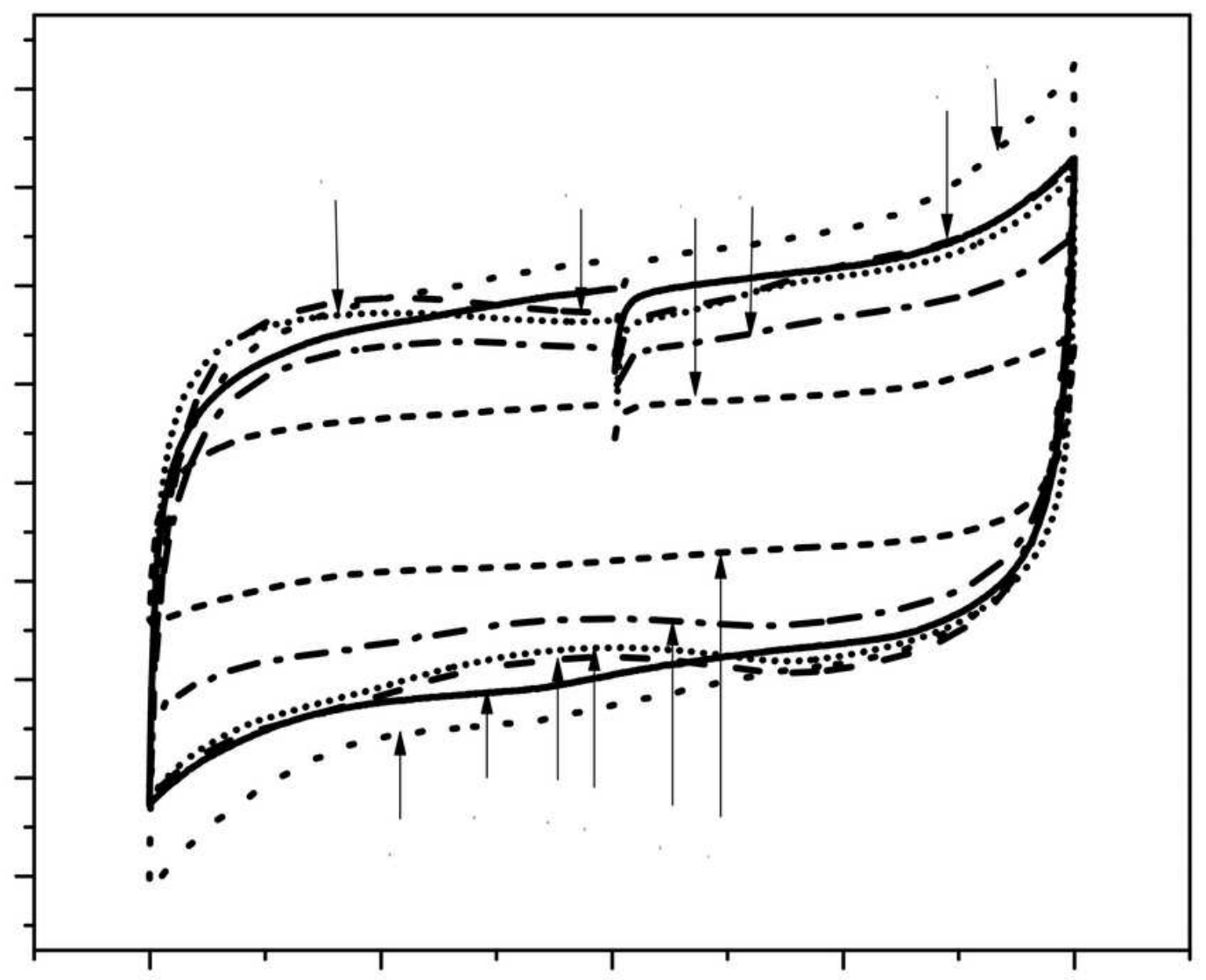

Figure 4

Cyclic voltammograms of EDLCs having EG electrodes with different exfoliation time. Potential window of $-1.0 \mathrm{~V}$ to $+1.0 \mathrm{~V}$ and scan rate $10 \mathrm{mV} \mathrm{s}-1$. [(1) Graphite, (2) $45 \mathrm{~min} \mathrm{EG}$, (3) $2 \mathrm{~h} \mathrm{EG,} \mathrm{(4)} 5 \mathrm{~h} \mathrm{EG,} \mathrm{(5)} 10 \mathrm{~h}$ EG, (6) 15 h EG] 


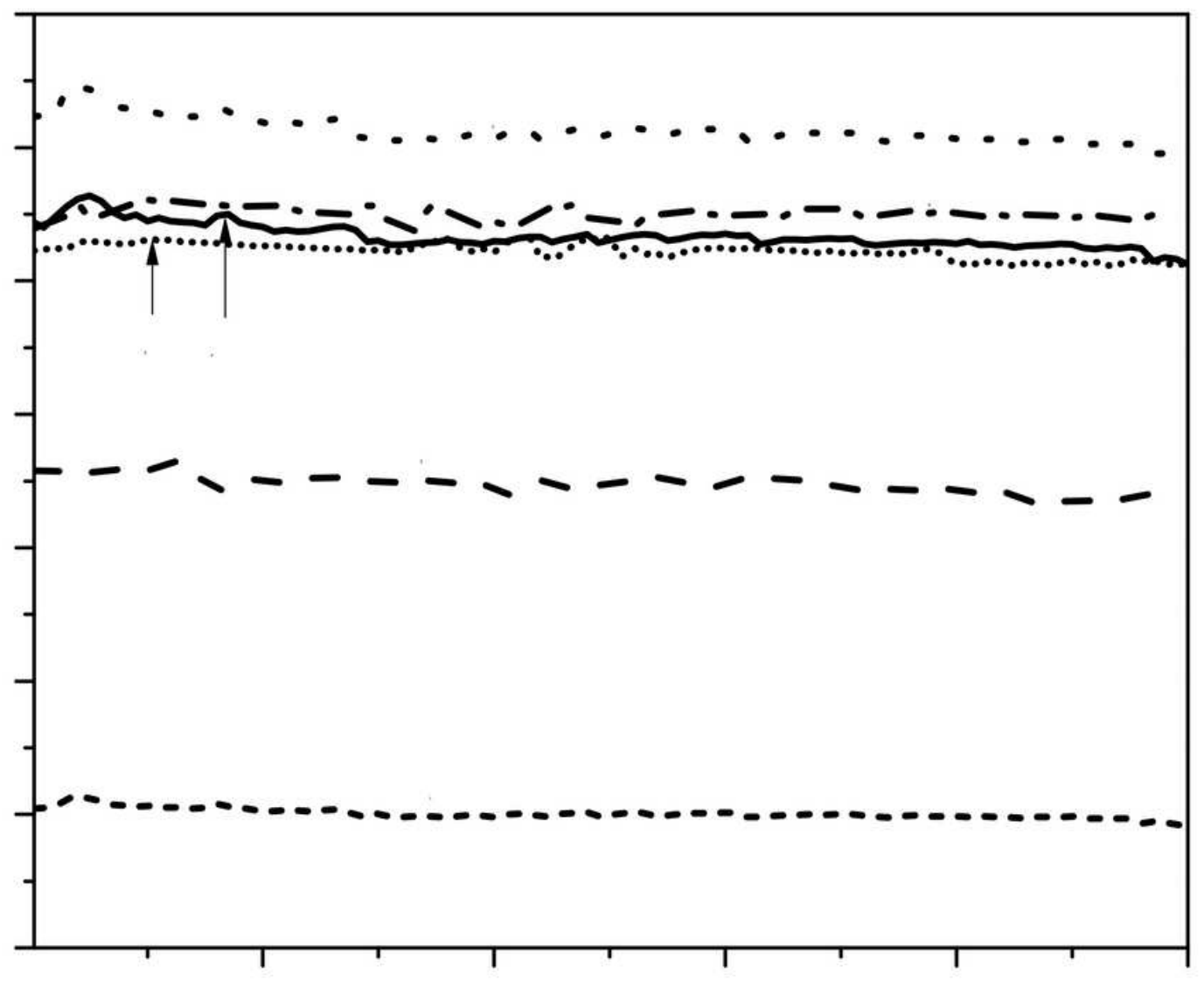

Figure 5

Single electrode specific capacitance (Csc) values obtained from continuous cycling over 500 cycles for EDLC with different EG electrodes. [(1) Graphite, (2) 45 min EG, (3) 2 h EG, (4) 5 h EG, (5) 10 h EG, (6) 15 h EG] 


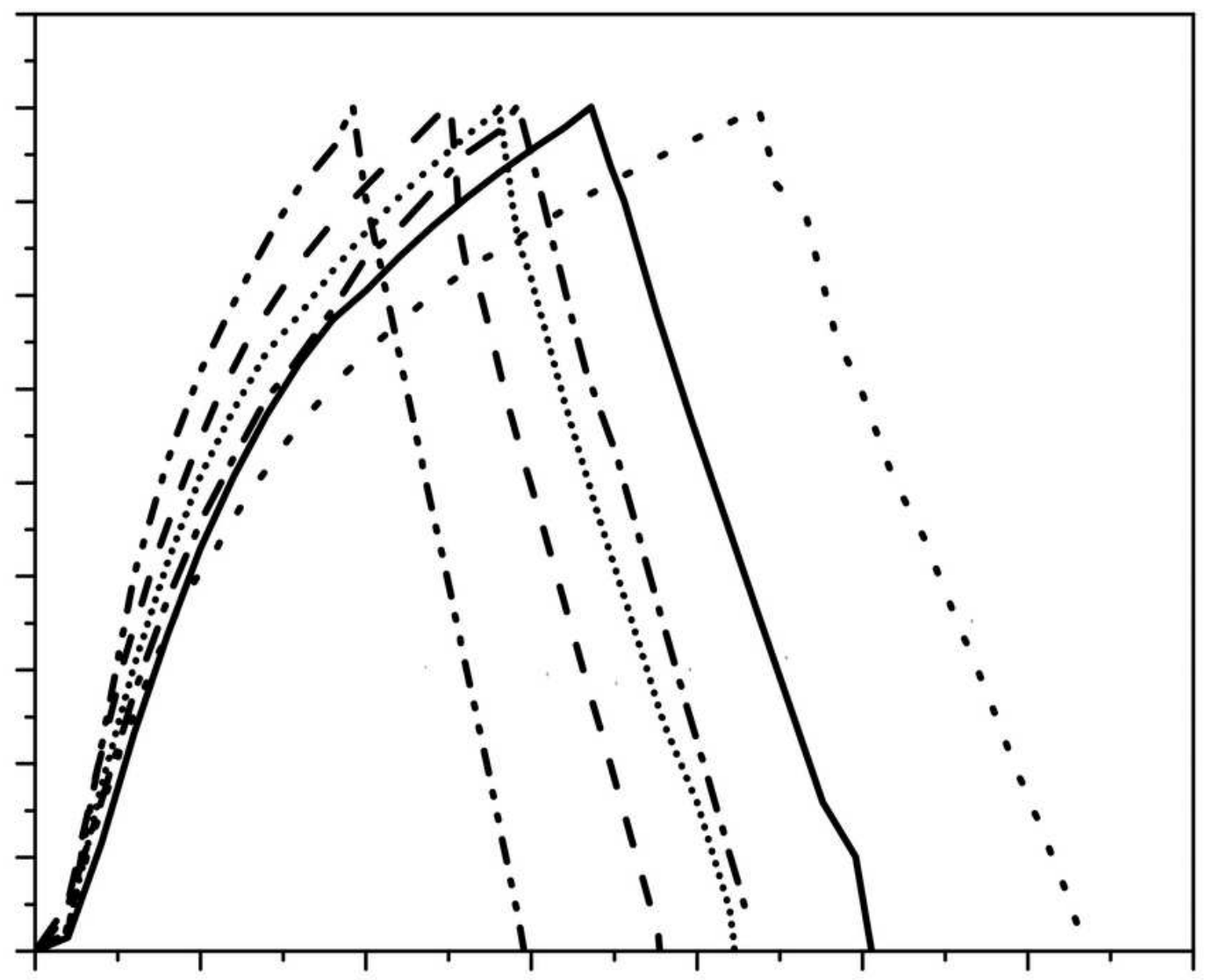

Figure 6

Initial charging and discharging cycles of different EG samples obtained in the + $0.1 \mathrm{~V}$ to $+1.0 \mathrm{~V}$ potential range. [(1) Graphite, (2) 45 min EG, (3) 2 h EG, (4) 5 h EG, (5) 10 h EG, (6) 15 h EG] 


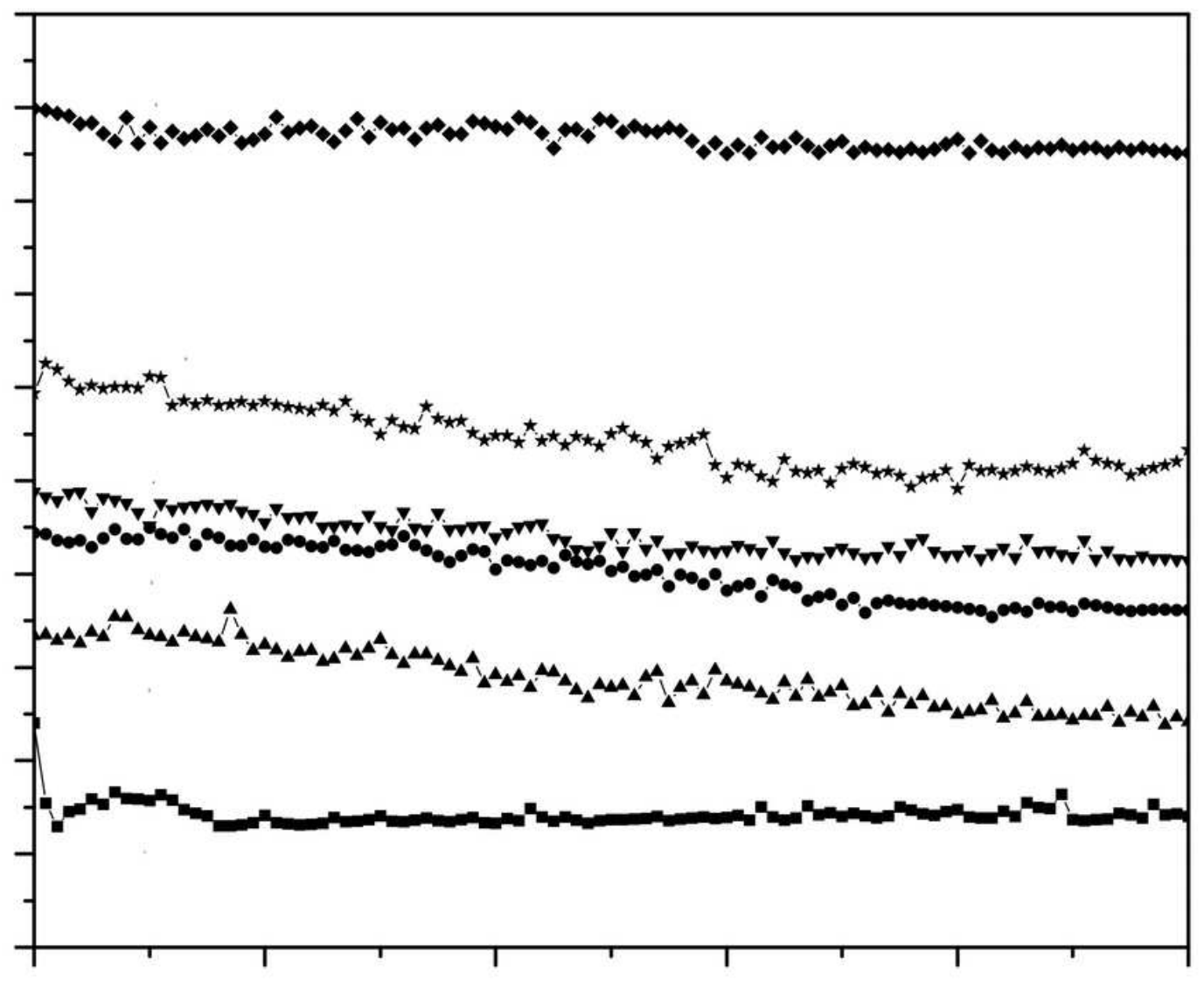

Figure 7

Single electrode discharge capacitance (Csd) values obtained from continuous charge-discharge cycling for 10000 cycles for different EG samples. [(1) Graphite, (2) 45 min EG, (3) 2 h EG, (4) 5 h EG, (5) 10 h EG, (6) $15 \mathrm{~h} \mathrm{EG]}$ 